\title{
Éduquer à la confiance dans une société de défiance
}

Le cas de la France

Instilling trust through education in a distrustful society. The case of France

Educar a la confianza en una sociedad de desconfianza. El caso de Francia

Denis Meuret

\section{OpenEdition}

Journals

Édition électronique

URL : https://journals.openedition.org/ries/5509

DOI : 10.4000/ries.5509

ISSN : 2261-4265

Éditeur

France Education international

Édition imprimée

Date de publication : 1 septembre 2016

Pagination : 67-76

ISBN : 978-2-85420-611-1

ISSN : $1254-4590$

Référence électronique

Denis Meuret, "Éduquer à la confiance dans une société de défiance », Revue internationale d'éducation de Sèvres [En ligne], 72 I septembre 2016, mis en ligne le 01 septembre 2018, consulté le 01 juillet 2021. URL : http://journals.openedition.org/ries/5509 ; DOI : https://doi.org/10.4000/ries.5509

(c) Tous droits réservés 


\title{
Éduquer à la confiance dans une société de défiance
}

\section{Le cas de la France}

\author{
Denis Meuret \\ Université de Bourgogne-Franche-Comté, \\ IREDU
}

Il y a plusieurs raisons de s'interroger sur la place de la confiance dans l'école française.

La première est que la confiance est un bien public de plus en plus important dans les sociétés modernes, dans lesquelles les interactions avec des personnes inconnues ou peu connues se multiplient.

En comparant les relations entre les performances économiques et les attitudes sociales dans une trentaine de pays du début des années 50 à nos jours, nous constatons que le déficit de confiance et d'esprit civique réduit significativement et durablement le revenu par habitant. (Algan et Cahuc, 2008)

Algan et Cahuc montrent aussi que les comportements inciviques, fréquents en France, ont un lien avec la défiance. Par ailleurs, le bon sens suggère que la confiance en l'autre favorise le débat, l'expression des idées et, par là, la démocratie, tandis que le populisme fait son lit de la défiance. On peut en déduire que la confiance est l'une des qualités que l'école doit s'efforcer de développer.

Ceci d'autant plus, semble-t-il, que selon les études internationales, les adultes français sont parmi ceux qui font le moins confiance à autrui ou à leurs institutions. Ainsi, dans l'enquête OCDE sur la littératie des adultes (PIAAC), qui comportait deux questions sur la confiance que l'on peut faire à autrui, 10,3\% seulement des répondants français étaient en désaccord avec l'idée qu' « il y a seulement un petit nombre de personnes à qui on peut faire entièrement confiance »; ce pourcentage est deux fois moindre que la moyenne des 19 pays de l'enquête PIAAC, il est bien plus proche de celui du pays le plus "méfiant" de ces pays (l'Italie, avec $7 \%$ ) que de celui du plus " confiant» (le Danemark avec 44,9\%). Par ailleurs, 14,2\% seulement des répondants français étaient en désaccord avec l'idée que "si vous ne faites pas attention, les autres profiteront de vous ", alors que la moyenne PIAAC est de $17,7 \%$ et que la proportion de confiants est de 41,6 \% dans le pays le plus confiant selon cet indicateur (la Suède) et de $5 \%$ dans le pays le moins confiant (la République tchèque) (Bongonovi et Burns, 2015). Algan et Cahuc (2008) citent d'autres chiffres plus anciens mais également impressionnants. 
La seconde raison de s'interroger sur la place de la confiance dans l'école française est que, comme d'autres organisations, l'école fonctionne mieux si ses acteurs (élèves, enseignants, parents, chefs d'établissement, etc.) se font confiance. D’une façon générale, les élèves obéiront plus volontiers à des enseignants à qui ils font confiance pour vouloir le mieux pour eux, pour leur demander des efforts utiles. En ce sens, la confiance est sans doute, davantage que le trop célèbre respect, une condition nécessaire de l'autorité. Plus spécifiquement, des élèves confiants en leurs enseignants, des enseignants confiants en leurs collègues ou en leur chef d'établissement, leur feront part plus facilement et plus honnêtement de leurs difficultés, les préviendront plus facilement s'ils les voient emprunter une voie sans issue; des parents confiants accepteront plus facilement des innovations ; des professeurs confiants dans leur chef d'établissement ou dans l'administration accepteront plus facilement de nouvelles politiques, accepteront plus facilement qu'elles ne produisent leurs bénéfices qu'après une période de réglage à laquelle ils se prêteront plus volontiers. Seul un petit nombre de recherches empiriques existe (aux États-Unis) sur la confiance à l'école et ses effets. Elles montrent, d'une part, que le degré de confiance des catégories d'acteurs de l'école les unes vis-à-vis des autres varie beaucoup d'une école à l'autre et, d'autre part, que, sous contrôle des variables sociodémographiques habituelles, le degré de confiance est lié positivement à l'apprentissage des élèves (Goddard et al., 2001), et à la mise en œuvre de réformes (Bryk et Schneider, 2003).

On voit cependant que ces deux raisons posent deux questions différentes à l'école : "quelle contribution peut-elle apporter au développement de la confiance dans la société » et "comment peut-elle développer la confiance entre ses acteurs pour que les élèves apprennent mieux?» Ces deux questions sont liées : c'est une même expérience scolaire qui doit à la fois développer la confiance au sein de l'école et favoriser la confiance sociale dans une société gangrenée par la méfiance. Pour le dire autrement, l'école doit être un lieu où un certain exercice de la confiance à la fois permet un meilleur apprentissage et favorise un usage éclairé de la confiance dans une société de défiance. Pour mieux discerner ce que pourrait être une telle expérience, il faut faire un détour par ce que nous savons des sources de la méfiance.

\section{LES SOURCES DE LA MÉFIANCE}

Les chercheurs définissent la confiance comme une disposition à s'exposer à un comportement négatif de l'autre partie de l'échange. Ils distinguent la confiance dans les institutions et la confiance interpersonnelle, elle-même pouvant être "personnalisée » (envers ses amis, sa famille) ou « sociale» (envers des inconnus) (Bongonovi et Burns, 2015). La "confiance sociale » est « le fait de s'attendre à ce que les autres membres de la communauté se comportent de façon coopérative et honnête ». Une forte confiance personnalisée peut aller de pair avec une faible confiance sociale (c'est l'un des symptômes du communautarisme : seuls les membres du groupe sont dignes de confiance). 
Certaines approches de la confiance en font une sorte de disposition psychologique (comme par exemple Bongonovi et Burns, 2015). À ce compte, l'affaire est simple : cette disposition ayant de bons effets sociaux, l'école doit la développer chez les élèves, il convient seulement de savoir quel type d'école (ou de société) la développe le mieux. Ainsi, Bongonovi et Burns, sur la base des données de PIAAC 2012 ${ }^{1}$, trouvent des corrélations entre la disposition à faire confiance et la faiblesse des inégalités sociales dans une société donnée. Elles trouvent aussi que les individus qui ont fait de longues études, les enfants de parents éduqués, ceux qui ont les meilleures compétences en lecture et écriture (ceci, estiment-elles, parce que ces deux activités «entraînent la capacité à se mettre dans la peau de quelqu'un d'autre ») et ceux qui occupent des positions sociales élevées sont plus enclins à faire confiance.

Par ailleurs, on a trouvé des origines... biologiques à la propension à faire confiance: Tirole (2016) relate qu'une expérience a montré que la propension à la confiance augmentait chez les individus après une injection d'ocytocyne.

Mais, d'un autre côté, on peut estimer que la disposition à faire confiance procède surtout de l'expérience : plus l'individu peut éprouver ou observer des situations où sa confiance ou celle de quiconque a été déçue, moins il sera enclin à accorder sa confiance.

La confiance n'est pas une orientation affective envers les autres mais répond à la fiabilité [trustworthyness] des différents candidats à votre confiance. (Forsyth, 2008, traduction D. Meuret).

On peut observer, à l'appui de cette assertion, que les pays où les adultes déclarent le plus (le moins) souvent faire confiance aux autres sont aussi les pays dont l'indice de non-corruption établi par l'association Transparency International est le plus (le moins) élevé (voir l'annexe 1). Sur les 19 pays de l'enquête PIAAC, la corrélation entre l'indice de confiance (la proportion de répondants qui ne sont pas d'accord avec l'affirmation qu'on peut complètement faire confiance à seulement un petit nombre de personnes) et l'indice de noncorruption de Transparency International est de 0,82 (calcul de l'auteur). Dans les pays nordiques, la confiance est élevée et la corruption faible, dans les pays latins (Espagne, Italie, France à un degré moindre), la confiance est plus faible et la corruption plus forte. Algan et Cahuc (2008) mettent en évidence, sur des données plus anciennes, un lien semblable entre indice de méfiance et indice de corruption. Autrement dit, les individus sont plus enclins à la confiance dans les pays où il est en effet davantage possible de faire confiance.

1. PIAAC est l'évaluation OCDE des compétences des adultes. Elle mesure leurs compétences en lecture et écriture, mais comprend aussi des indications sur la position sociale et les études de l'individu et de ses parents. La disposition à (ne pas) faire confiance est mesurée par le degré de désaccord (ou d'accord) avec deux affirmations : « ifyou are not careful, other people will take advantage of you » [si vous ne faites pas attention, d'autres personnes vont profiter de vous] et " there are only a few people you can trust completely " [il y a seulement un petit nombre de personnes à qui on peut faire entièrement confiance]. 
Cependant, le degré de confiance en France (10,3) est environ deux fois plus faible que prédit (environ 20) par la perception de la corruption (calcul de l'auteur). Même si d'autres expériences que celle de la corruption peuvent miner la confiance, on peut penser que d'autres causes que l'expérience jouent négativement en France. Algan et Cahuc (2008) incriminent, à partir d'études empiriques convaincantes, le modèle français d'État-providence (corporatiste et étatiste) qui s'est mis en place en France à la Libération. Par exemple, celui-ci lie davantage que d'autres les prestations au statut de chacun, ce qui génère le soupçon de statuts privilégiés, suscite ressentiment et méfiance. Leur étiologie de la méfiance ne fait pas de place à telle ou telle caractéristique de l'école française.

Il ne résulte néanmoins pas de l'étiologie proposée par ces auteurs que l'école ne joue aucun rôle dans le surcroît de méfiance que semblent manifester les Français par rapport aux raisons objectives qu'ils ont d'être méfiants. Il est même difficile de croire qu'une expérience si durable puisse ne pas laisser de traces. Partons donc de l'idée que l'expérience scolaire détermine à la fois la confiance au sein de l'école et, partiellement, la confiance sociale.

Nous devons donc rechercher les formes d'une expérience qui à la fois favorise la première et éduque à faire confiance, dans une société où il peut arriver qu'elle soit déçue.

\section{UNE EXPÉRIENCE SCOLAIRE QUI FAVORISE LA CONFIANCE AU SEIN DE L'ÉCOLE}

La théorie économique contemporaine explique fort bien que les acteurs de l'école ont de bonnes raisons de ne pas se faire confiance. L'apprentissage, en effet demande la contribution de plusieurs acteurs (l'élève et l'enseignant au premier chef) et ces acteurs n'ont qu'une information imparfaite sur les efforts des autres, de sorte que chacun peut suspecter l'autre de ne pas faire assez d'effort et de saboter les siens propres. Nous avons tous en tête des exemples multiples de ce type de méfiance, y compris des acteurs de terrain vis-à-vis de l'administration. Cependant, nous avons en réalité peu de données rigoureuses sur le sujet. Les évaluations internationales, à ma connaissance, ne posent pas de questions sur le degré de confiance que les acteurs éprouvent les uns vis-àvis des autres.

Il y a quelques raisons de penser que les acteurs de l'école française se font plutôt moins confiance que ceux d'autres écoles. Il fait partie, en effet, du modèle sur lequel repose la tradition scolaire française, disons l'école républicaine (Meuret, 2007, 2013) que l'école agit contre les tendances profondes des élèves et de la société, donc des parents, de sorte que les enseignants (et les chefs d'établissement) sont, plus qu'ailleurs, fondés à se méfier des premiers et de 
penser que la seconde ne les aime pas $^{2}$. Inversement, les modèles dominants en Amérique du Nord (États-Unis, Canada), ceux d'une école au service de la société ou de la communauté, favorisent sans doute davantage la confiance entre les acteurs de l'école. Je n'ai pas trouvé de données sur la confiance des acteurs de l'école les uns dans les autres permettant de tester cette affirmation ${ }^{3}$. Pour ce faire néanmoins, je propose une liste de facteurs susceptibles de favoriser la confiance entre les acteurs de l'école, dont la prévalence peut être vérifiée à travers les enquêtes internationales.

Disons qu'une école qui crée un climat de confiance entre ses acteurs pourrait ressembler à ceci :

1) une école qui fait son travail, en particulier faire apprendre les élèves : on ne peut faire confiance à quelqu'un qui ne tient pas la promesse implicite dans la relation qui le lie à vous ;

2) une école qui réduit au minimum la violence entre ses acteurs, les protège les uns des autres : on ne peut faire confiance ni à quelqu'un qui vous harcèle ni à quelqu'un qui vous laisse harceler ;

3) une école où les élèves ont le sentiment d'être traités avec justice ;

4) une école qui dit vrai : contre la tentation de prêcher aux élèves des comportements vertueux, François Dubet (2016) écrit justement : «Comment croire que les élèves auront toujours confiance dans des leçons que leur expérience dément souvent?»;

5) une école qui fait confiance, donne aux enseignants et aux élèves des libertés et des responsabilités : comment faire confiance à une école qui prêcherait la confiance, mais ne ferait pas elle-même confiance à ses acteurs (professeurs et élèves) ? ;

6) une école transparente, qui accepte de rendre des comptes: on ne fait plus confiance aujourd'hui à qui réclame votre confiance sans donner aucun moyen de juger de son bien-fondé ; on fait davantage confiance à qui se reconnaît faillible et responsable.

Certaines enquêtes internationales posent aux élèves (PISA) ou aux enseignants (TALIS) des questions qui correspondent, plus ou moins bien il est vrai, à ces dimensions. Nous proposons en annexe 2 une comparaison de l'école française avec les écoles états-unienne et canadienne, comparaison fort fruste évidemment (il faudrait avoir davantage de critères, mieux étayés, et distinguer entre les différentes catégories d'acteurs), mais qui nous paraît la moins mauvaise, compte tenu des données disponibles.

2. Selon l'enquête TALIS, $5 \%$ des enseignants français estiment leur métier valorisé par la société, contre $31 \%$ de moyenne internationale.

3. Ce que nous pouvons savoir sur la situation française est, par des sondages, qu'une petite majorité de Français fait confiance à l'école en général, davantage à l'école primaire qu'à l'école secondaire ; et par une enquête (Fotinos, 2014), que la défiance est grande et réciproque entre les directeurs d'école et les parents. 
Ces données ne témoignent pas d'une distance abyssale entre les écoles correspondant aux deux modèles, mais elles vont dans le sens attendu : le plus souvent ${ }^{4}$, les facteurs facilitant la confiance sont davantage présents en Amérique du Nord qu'en France : les acteurs estiment plus fréquemment là-bas que l'école fait son travail $\left(+17\right.$ points $\left.^{5}\right)$, que les élèves sont traités avec justice $(+21)$, les enseignants disent plus fréquemment s'efforcer de lier leur enseignement à l'expérience quotidienne des élèves $(+15)$, que l'école fait participer élèves $(+13)$ et parents $(+8)$ aux décisions. Enfin, l'école démocratique rend davantage compte de ses résultats au public $(+27)$. Sur la question de la prévention de la violence, les écarts sont moins clairs, parfois en faveur des écoles d'Amérique du Nord, parfois de la France.

Les données, imparfaites, dont nous disposons vont donc plutôt dans le sens de l'idée que les acteurs de l'école se font moins confiance en France qu'ailleurs et que, pour développer la confiance en son sein, l'école française pourrait s'inspirer de ce qui se passe de l'autre côté de l'Atlantique.

\section{UNE EXPÉRIENCE SCOLAIRE QUI ÉDUQUE À LA CONFIANCE}

L'école est, par construction, un milieu protégé au sein duquel il est vital que l'élève puisse faire confiance à ses interlocuteurs (autres élèves, enseignants, etc.). En même temps, elle doit le préparer à interagir dans un monde où la confiance est souhaitable à cause de ses bons effets collectifs, en même temps qu'il est souhaitable de ne pas faire confiance indûment. Comment gérer cette tension? Sans doute d'abord dans le temps : la protection doit être maximale pour les jeunes enfants, en particulier à l'école primaire. C'est après qu'il faut laisser émerger progressivement la question de l'opportunité de faire confiance.

Sans doute l'école doit-elle alors envoyer trois types de messages aux élèves :

1) Le monde n'est pas parfait. On ne peut pas faire confiance à tout le monde dans n'importe quelle circonstance. Bien sûr, l'histoire procure grand nombre d'exemples de confiance trahie et cet aspect peut être souligné, mais cela peut être dit aussi à travers, par exemple, des discussions sur des affaires de corruption ou d'accusations qui se sont avérées fausses, ou encore sur la confiance qu'on peut faire aux réponses aux enquêtes, y compris celles que les élèves peuvent faire eux-mêmes (l'analyse de ces enquêtes doit intégrer la question de la fiabilité des réponses et des raisons pour lesquelles des réponses peuvent être

4. Pas toujours cependant : il arrive que les écoles canadiennes et françaises soient plus proches entre elles que chacune des États-Unis (transparence), que les écoles états-unienne et française soient plus proches entre elles que chacune du Canada (participation des élèves aux décisions), que les trois écoles soient très proches (fréquence des écoles primaires sans problèmes de discipline)

5. On donne la moyenne pour les indicateurs du facteur, de la valeur (USA + Canada)/2 - F. 
insincères). Le travail en groupe, la coopération de façon plus générale sont sûrement un moyen de développer la confiance, puisqu'on y découvre à la fois l'intérêt de la coopération et qu'il faut se faire confiance pour coopérer. Cependant, la construction des situations de coopération doit sans doute évoquer avec les élèves - et non refouler - la possibilité que puissent s'y produire des abus de confiance, par exemple des "passagers clandestins », comme disent les économistes, qui profitent du travail des autres membres du groupe.

2) A priori, cependant, il vaut mieux faire confiance. On peut dire les choses ainsi : accorder sa confiance est exposé et à des erreurs de type 1 (on fait confiance à quelqu'un qui ne le mérite pas, on ne se méfie pas assez) et à des erreurs de type 2 (on refuse à tort sa confiance, on se méfie trop). L'éducation à la confiance consisterait dès lors à faire prendre conscience qu'on risque un désavantage si on ne se méfie pas assez (erreur de type 1) mais aussi si on se méfie trop (erreur de type 2). En réalité, on risque davantage une erreur de type 2 que de type 1 : en économie expérimentale, le "jeu de la confiance» établit qu'en moyenne, les joueurs aveugles à la personnalité de l'autre lui font plus souvent confiance qu'ils ne s'en méfient et qu'ils ont raison de ce comporter ainsi (Tirole, 2016). Le simple bon sens montre d'ailleurs qu'aucune forme de coopération sociale ne serait possible sans un minimum de confiance, ce pourquoi, d'ailleurs, trahir la confiance d'autrui est si délétère et répréhensible.

3) Il faut accorder sa confiance à bon escient. Le rôle de l'école est sans doute ici de montrer aux élèves que le critère pertinent pour décider si l'on fait ou non confiance est la situation plus que la catégorie à laquelle appartient le partenaire. Par exemple, il est davantage possible de faire confiance quand les protagonistes sont appelés à se revoir et que leur comportement engage leur réputation, quand l'autre est conscient que vous pouvez lui retirer la confiance que vous lui accordez et qu'il peut y perdre.

On peut synthétise cela en proposant à l'école non pas de prêcher la confiance, mais de mettre en place des situations qui montrent aux élèves l'intérêt de se faire confiance ainsi que la possibilité que la confiance soit trahie, et de travailler avec eux les leçons à tirer de ces expériences.

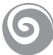

Cette analyse met en lumière le fait que les acteurs et les partenaires de l'école se font probablement moins confiance en France que dans des pays proposant un autre modèle d'école et que la défiance acquise par l'expérience scolaire peut expliquer que celle-ci soit, au sein de la société française, plus répandue encore que ne le voudrait le niveau de corruption, non négligeable, qui y règne. Elle conduit donc à distinguer deux enjeux pour l'école française. D'une part aller vers un fonctionnement qui favorise davantage la confiance entre ses acteurs. D'autre part, proposer aux élèves une expérience qui les éduque à la confiance, ce qui signifie, dans le cadre d'une société française méfiante en partie à raison, 
avertir les élèves de ce que les avantages de faire confiance à autrui sont dans l'ensemble nettement plus grands que les risques que cette attitude fait courir, mais aussi de ce que la confiance peut être déçue et leur proposer des expériences qui les aident à discerner les risques et à faire confiance à bon escient.

\section{BibLIOGRAPHIE}

ALGANY CAHUC P. (2008) : La société de défiance, Paris : éditions rue d'Ulm.

BORGONOVI F., BURNS T. (2015): "The Educational Roots of Trust», OCDE Working Papers, $\mathrm{n}^{\circ} 119$.

BRYK A.S., SCHNEIDER B. (2003) : " Trust in school, a core resource for school reform ", Educational Leadership, 60 (6).

FORSYTHP B. (2008) : «The empirical consequences of school trust», in W.H. Hoy et M. DiPaola (dir.), Improving Schools Studies in leadership and culture, Charlotte NC : Information Age Publishing.

GODDARD R.D. et al. (2001) : "A multilevel examination of the distribution and effects of teacher trust in students and parents in Urban Elementary Schools", The Elementary School Journal, 102 (1).

FOTINOS G. (2014) : Entre méfiance, confiance et bienveillance, CASDEN/Espé de Lyon/UCLB Lyon/UNIRES [en ligne] : [http://goo.gl/eft6ny]

MEURET D. (2013) : Pour une école qui aime le monde, PUR (Paideia - éducation, savoir, société).

MEURET D. (2007) : Gouverner l'école, PUF (Éducation et société).

SCHUTZ A. (2011) : " Power and Trust in the Public Realm: John Dewey, Saul Alinski and the limits of Progressive Education », Educational Theory, 61 (4).

TIROLE J. (2016) : Économie du bien commun, PUF. 
ANNEXE 1.

Confiance sociale entre les adultes et corruption, une comparaison entre pays

\begin{tabular}{|l|c|c|}
\hline Pays & Indice de non corruption (2012) & Indice de confiance sociale (2012) \\
\hline Danemark & 90 & 44,9 \\
\hline Finlande & 90 & 33,3 \\
\hline Suède & 88 & 33,7 \\
\hline Pays-Bas & 84 & 31,4 \\
\hline Norvège & 85 & 34,0 \\
\hline Canada & 84 & 25,0 \\
\hline Allemagne & 79 & 14,9 \\
\hline Royaume Uni (Angleterre) & 74 & $(18,7)$ \\
\hline Australie & 85 & 23,0 \\
\hline Autriche & 69 & 22,9 \\
\hline États-Unis & 73 & 22,3 \\
\hline Irlande & 69 & 16,0 \\
\hline Japon & 74 & 18,2 \\
\hline France & 71 & 10,3 \\
\hline Pologne & 58 & 15,5 \\
\hline Espagne & 56 & 5,0 \\
\hline République tchèque & 42 & 10,6 \\
\hline Corée du Sud & 56 & \\
\hline Italie & 59 & \\
\hline
\end{tabular}

Lecture : Nous appelons « indice de non-corruption » l'Index of Perceived Corruption calculé par l'ONG Transparency International, indice qui va de o pour une corruption très élevée à 100 pour une absence de corruption (ce pourquoi nous préférons l'appeler « indice de non-corruption »); selon cette organisation, cet indice " permet de saisir les perceptions de l'étendue de la corruption dans le secteur public du point de vue des hommes et femmes d'affaire et des experts des pays »; « il s'appuie sur des sources de données provenant d'institutions indépendantes spécialisées dansl'analyse de lagouvernance et du climat desaffaires ».Voir : [https://www.transparency.org/cpi2015/\#downloads], puis cliquer sur FAQ. On trouve cet indice pour 160 pays à l'adresse : [www.transparency.org/CPI2015]. Pour une meilleure comparaison avec les données de PIAAC, nous utilisons la valeur de cet indicateur pour 2012.

Nous appelons « indice de confiance sociale » la proportion de personnes qui ont répondu, lors de l'enquête de L'OCDE sur la littératie des adultes (PIAAC, 2012), être en désaccord ou tout à fait en désaccord avec l'affirmation " there are only a few people you can trust completely " [il y a seulement un petit nombre de personnes à qui on peut faire entièrement confiance]. Cet indice est reporté d'après Bongonovi et Burns (2015), pour les 19 pays de l'enquête PIAAC.

Les pays pris en compte sont ceux dont les données sont disponibles pour les deux indices. Ils sont classés par ordre décroissant de la valeur de leur indice de non-corruption en 2015. 
ANNEXE 2.

Facteurs de confiance dans l'école « démocratique " (États-Unis, Canada) et l'école " républicaine » (France), selon PISA 2012 (élèves de 15 ans), PIRLS 2011 (élèves de CM1) et TALIS 2013 (enseignants de collèges)

\begin{tabular}{|c|c|c|c|c|c|}
\hline $\begin{array}{l}\text { Facteurs : } \\
\text { l'école... }\end{array}$ & Question posée & France & $\begin{array}{l}\text { États- } \\
\text { Unis }\end{array}$ & Canada & $\begin{array}{c}(\mathrm{US}+\mathrm{C}) / \\
2-\mathrm{F}\end{array}$ \\
\hline \multirow{3}{*}{$\begin{array}{l}\text {.. fait } \\
\text { son travail }\end{array}$} & $\begin{array}{l}\text { Les professeurs montrent de l'intérêt pour } \\
\text { l'apprentissage de tous les élèves (PISA). }\end{array}$ & $50 \%$ & $69 \%$ & $67 \%$ & 18 \\
\hline & $\begin{array}{l}\text { Le professeur maintient l'ordre en classe } \\
\text { (PISA). }\end{array}$ & $67 \%$ & $82 \%$ & $81 \%$ & 14 \\
\hline & $\begin{array}{l}\text { Selon les enseignants, l'école accorde une } \\
\text { très grande importance à la réussite scolaire } \\
\text { des élèves (PIRLS). }\end{array}$ & $59 \%$ & $79 \%$ & $78 \%$ & 19 \\
\hline \multirow{6}{*}{$\begin{array}{l}\ldots \text { prévient } \\
\text { la violence }\end{array}$} & $\begin{array}{l}\% \text { d'enseignants dont le chef d'établissement } \\
\text { dit observer plus d'une fois par semaine : } \\
\text {.. des intimidations ou agressions verbales } \\
\text { d'élèves (TALIS). }\end{array}$ & 23,7 & 20,9 & $28,7^{*}$ & +1 \\
\hline & $\begin{array}{l}\ldots \text { des violences significatives entre élèves } \\
\text { (TALIS). }\end{array}$ & 7,0 & 1,7 & $2,0^{*}$ & -5 \\
\hline & $\begin{array}{l}\text {... des intimidations ou agressions verbales } \\
\text { contre des enseignants ou } \\
\text { d'autres membres du personnel (TALIS). }\end{array}$ & 3,0 & 4,7 & $2,6^{*}$ & +1 \\
\hline & $\begin{array}{l}\text { L'école est un endroit sûr et ordonné selon } \\
\text { ses enseignants (PIRLS). }\end{array}$ & 40 & 64 & 62 & +12 \\
\hline & $\begin{array}{l}\text { Élèves disant qu'ils ne sont presque jamais } \\
\text { harcelés ou agressés (PIRLS). }\end{array}$ & 54 & 52 & 44 & -6 \\
\hline & $\begin{array}{l}\text { \% d'élèves dont le principal dit qu'il n’y a pas } \\
\text { ou quasiment pas de problèmes de sécurité } \\
\text { ou de discipline dans son école (PIRLS). }\end{array}$ & 62 & 63 & 60 & 0 \\
\hline ... est juste & $\begin{array}{l}\% \text { d'élèves estimant que « la plupart de mes } \\
\text { enseignants me traitent avec justice» (PISA). }\end{array}$ & 66 & 87 & 88 & 21 \\
\hline ... dit vrai & $\begin{array}{l}\text { Je (enseignant) me réfère fréquemment } \\
\text { à un problème de la vie quotidienne } \\
\text { pour démontrer l'utilité des connaissances } \\
\text { acquises (TALIS). }\end{array}$ & 57 & 71 & $74^{*}$ & 15 \\
\hline \multirow{2}{*}{$\begin{array}{l}\ldots \text { fait } \\
\text { confiance }\end{array}$} & $\begin{array}{l}\text { Cette école donne aux élèves l'occasion } \\
\text { de participer à la prise de décision (PISA) }\end{array}$ & 53 & 56 & 76 & 13 \\
\hline & $\begin{array}{l}\text { Cette école donne aux parents l'occasion } \\
\text { de participer à la prise de décision (PISA). }\end{array}$ & 70 & 79 & 77 & 8 \\
\hline $\begin{array}{l}\text {... est } \\
\text { transparente }\end{array}$ & $\begin{array}{l}\text { \% d'enseignants estimant que } \\
\text { «dans mon école, les données sur la réussite } \\
\text { des élèves sont publiées (TALIS).» }\end{array}$ & 45 & 83 & $60^{*}$ & 27 \\
\hline
\end{tabular}

*Alberta seulement. 